International Journal of Modern Physics E

(C) World Scientific Publishing Company

\title{
PAIRING PROPERTIES OF SUPERHEAVY NUCLEI
}

\author{
A. STASZCZAK ${ }^{a}$, J. DOBACZEWSKI ${ }^{b, c, d}$, W. NAZAREWICZ ${ }^{b, c, d}$ \\ ${ }^{a}$ Department of Theoretical Physics, Institute of Physics, Maria Curie-Sktodowska University, \\ pl. M. Curie-Sktodowskiej 1, 20-031 Lublin, Poland \\ ${ }^{b}$ Department of Physics, University of Tennessee, Knoxville, TN 37996, Knoxville, USA \\ ${ }^{c}$ Physics Division, Oak Ridge National Laboratory, P.O.Box 2008, Oak Ridge, TN 37831, USA \\ ${ }^{d}$ Institute of Theoretical Physics, Warsaw University, ul. Hoża 69, Warsaw, Poland \\ Received (received date) \\ Revised (revised date)
}

\begin{abstract}
Pairing properties of even-even superheavy $N=184$ isotones are studied within the Skyrme-Hartree-Fock+BCS approach. In the particle-hole channel we take the Skyrme energy density functional SLy4, while in the particle-particle channel we employ the seniority pairing force and zero-range $\delta$-interactions with different forms of density dependence. We conclude that the calculated static fission trajectories weakly depend on the specific form of the $\delta$-pairing interaction. We also investigate the impact of triaxiality on the inner fission barrier and find a rather strong $Z$ dependence of the effect.
\end{abstract}

\section{Introduction}

In the almost fifty years since the phenomenon of superconductivity was brought into nuclear structure, $\frac{1|2| 3}{1}$ our knowledge of the nature of pairing correlations in nuclei is still unsatisfactory and many fundamental questions remain (see Refs. 41516 and papers quoted therein). For instance, some components of the pairing interaction are believed to be induced, like in solid-state superconductors, ${ }^{7}$ some are directly rooted in the nucleon-nucleon force. In practical calculations based on effective interactions, one considers various pairing parametrizations. The resulting pairing fields have a strong influence on most low-energy properties of the nuclei 8 and the nuclear large-amplitude collective motion. Indeed, by increasing configuration mixing and reducing the magnitude of symmetry-breaking effects, pairing tends to make the nuclear collective motion more adiabatic, $\frac{9}{9}$ Therefore, when aiming at a quantitative understanding of fission properties of heavy and superheavy elements, it is important to have the pairing channel under control.

The purpose of this study is to compare different pairing schemes that are currently used in the Skyrme-Hartree-Fock+BCS (SHF+BCS) model to describe nuclear superfluidity. Within this framework, we compare the seniority paring force and state-dependent $\delta$-interaction (referred to as $\mathrm{SHF}+\mathrm{BCS}(\mathrm{G})$ and $\mathrm{SHF}+\mathrm{BCS}(\delta)$, respectively). As in our previous paper $\frac{10}{10}$ we focus here on the fission properties of even-even isotones with $N=184$; namely, we discuss the total binding energies, 
mass hexadecapole moments, and pairing gaps calculated along the static fission paths.

\section{Theoretical framework and results}

\subsection{Description of pairing correlations}

In our SHF+BCS approach, we use the Skyrme energy density functional in its SLy4 parameterization 11 in the particle-hole channel, whereas two different pairing schemes were implemented in the particle-particle channel. The $\mathrm{SHF}+\mathrm{BCS}(\mathrm{G})$ scheme employs the seniority pairing force with strength parameters defined as in Ref $\frac{12}{12}$, i.e.,

$$
\begin{aligned}
G^{n} & =[19.3-0.084(N-Z)] / A, \\
G^{p} & =[13.3+0.217(N-Z)] / A,
\end{aligned}
$$

additionally scaled by

$$
\tilde{G}^{n / p}=f_{n / p} G^{n / p}
$$

In the $\operatorname{SHF}+\operatorname{BCS}(\delta)$ scheme, we apply the state-dependent $\delta$-interaction 13 with commonly used parameterization variants, $\frac{14}{}$ which are summarized as

$$
V_{\delta}^{n / p}\left(\vec{r}_{1}, \vec{r}_{2}\right)=V_{0}^{n / p}\left[1-\eta\left(\frac{\rho\left(\frac{\vec{r}_{1}+\vec{r}_{2}}{2}\right)}{\rho_{0}}\right)\right] \delta\left(\vec{r}_{1}-\vec{r}_{2}\right),
$$

where $\rho_{0}=0.16 \mathrm{fm}^{-3}$ and

$\eta=\left\{\begin{array}{l}0, \text { for the delta interaction (DI), volume pairing; } \\ 1, \text { for the density-dependent delta interaction (DDDI), surface pairing; } \\ \frac{1}{2}, \text { for the mixed volume and surface pairing (MIX). }\end{array}\right.$

The scaling factors of Eq. (2),$f_{n}=1.41$ and $f_{p}=1.13$, and pairing strengths $V_{0}^{n}=282.0 \mathrm{MeV}, V_{0}^{p}=285.0 \mathrm{MeV}(\mathrm{DI}), V_{0}^{n}=842.0 \mathrm{MeV}, V_{0}^{p}=1020.0 \mathrm{MeV}$ (DDDI), and $V_{0}^{n}=425.5 \mathrm{MeV}, V_{0}^{p}=448.5 \mathrm{MeV}$ (MIX) were adjusted to reproduce the experimental 15 neutron $\left(\Delta_{n}=0.696 \mathrm{MeV}\right)$ and proton $\left(\Delta_{p}=0.803 \mathrm{MeV}\right)$ pairing gaps in ${ }^{252} \mathrm{Fm}$. As we deal with contact interactions, we use a finite pairingactive space defined by including $\Omega^{n / p}=(N$ or $Z)$ lowest single-particle states for neutrons and protons, respectively. In the $\mathrm{SHF}+\mathrm{BCS}(\delta)$ approach, the pairing gap is state dependent. Therefore, the average (spectral) gaps,

$$
\left\langle\Delta_{n / p}\right\rangle=\frac{\sum_{k \in \Omega^{n / p}} v_{k} u_{k} \Delta_{k}}{\sum_{k \in \Omega^{n / p}} v_{k} u_{k}},
$$

were used as measures of experimental pairing gaps deduced from the odd-even mass staggering. In Eq. (5) $v_{k}$ and $u_{k}$ are the BCS occupation amplitudes (see, e.g., Ref. $\frac{16}{16}$ for a more detailed discussion).

The calculations were carried out using the code HFODD (v.2.191) $17|18| 19$ that solves self-consistent HF equations by using a Cartesian 3D deformed harmonicoscillator finite basis. In the calculations, we took the lowest 1140 single-particle states for the basis. This corresponds to 17 oscillator shells at the spherical limit. 
A)

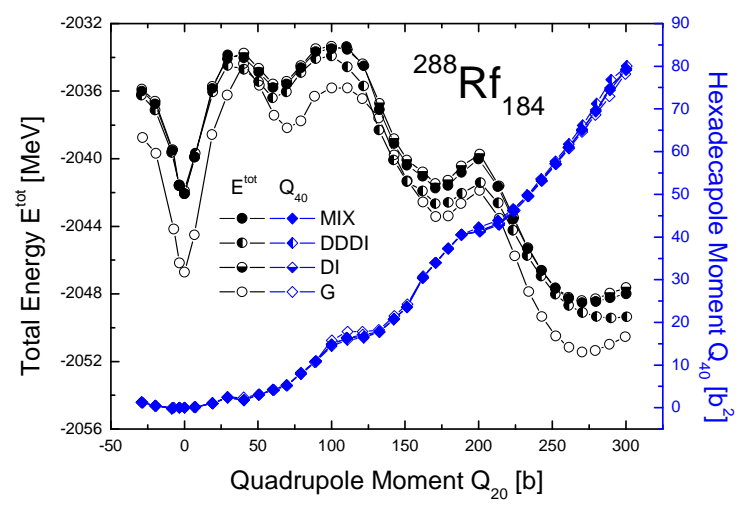

B)

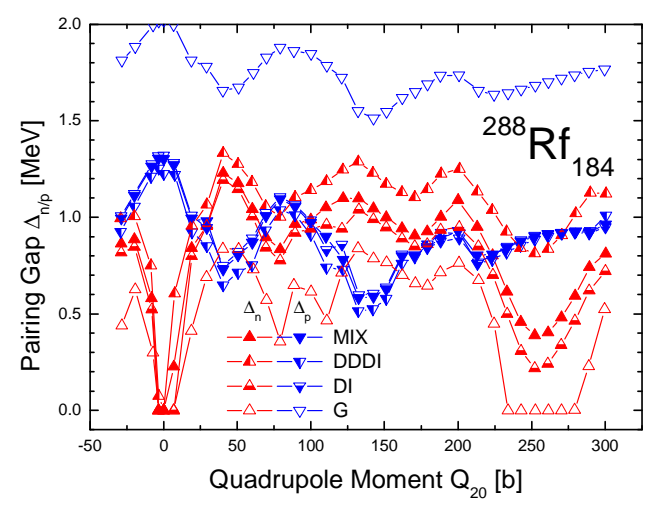

Fig. 1. (A) The total binding energies $E^{\text {tot }}$ (left-hand side scale) and mass hexadecapole moments $Q_{40}$ (right-hand side scale) along the fission paths of ${ }^{288} \mathrm{Rf}_{184}$ calculated with the SLy4 interaction and four different pairing interactions: MIX, DDDI, and DI $\delta$-interaction, and seniority pairing (G). (B) The neutron $\Delta_{n}$ and proton $\Delta_{p}$ pairing gaps along the fission paths shown above.

\subsection{Comparison of pairing models}

Figures 1, 2, and 3 display total binding energies $\left(E^{\text {tot }}\right)$, mass hexadecapole moments $\left(Q_{40}\right)$, and neutron/proton pairing gaps $\left(\Delta_{n / p}\right)$ calculated along the static fission paths of ${ }^{288} \mathrm{Rf}_{184},{ }^{298} 114_{184}$, and ${ }^{310} 126_{184}$. The fission paths were computed with a quadratic constraint 20 on the mass quadrupole moment $\left(Q_{20}\right)$. Our study covers the oblate/prolate deformations of $Q_{20}=-30 \div 300 \mathrm{~b}$ with a step of $10 \mathrm{~b}$.

As in previous studies based on the SLy4 functional, 2122 we found that all nuclei considered in this work have spherical shapes in their ground states. This is because $N=184$ appears to be the magic neutron number in most theoretical models based on the Skyrme approach. ${ }^{23}$ Due to the magic character of $N=184$ isotones, ground-state neutron pairing gaps calculated in BCS vanish. The groundstate proton pairing gaps, on the other hand, show considerable variations with $Z$. They are large in the open-shell rutherfordium $(Z=104)$, but in $Z=114$ the values 
4 A. Staszczak, J. Dobaczewski, W. Nazarewicz
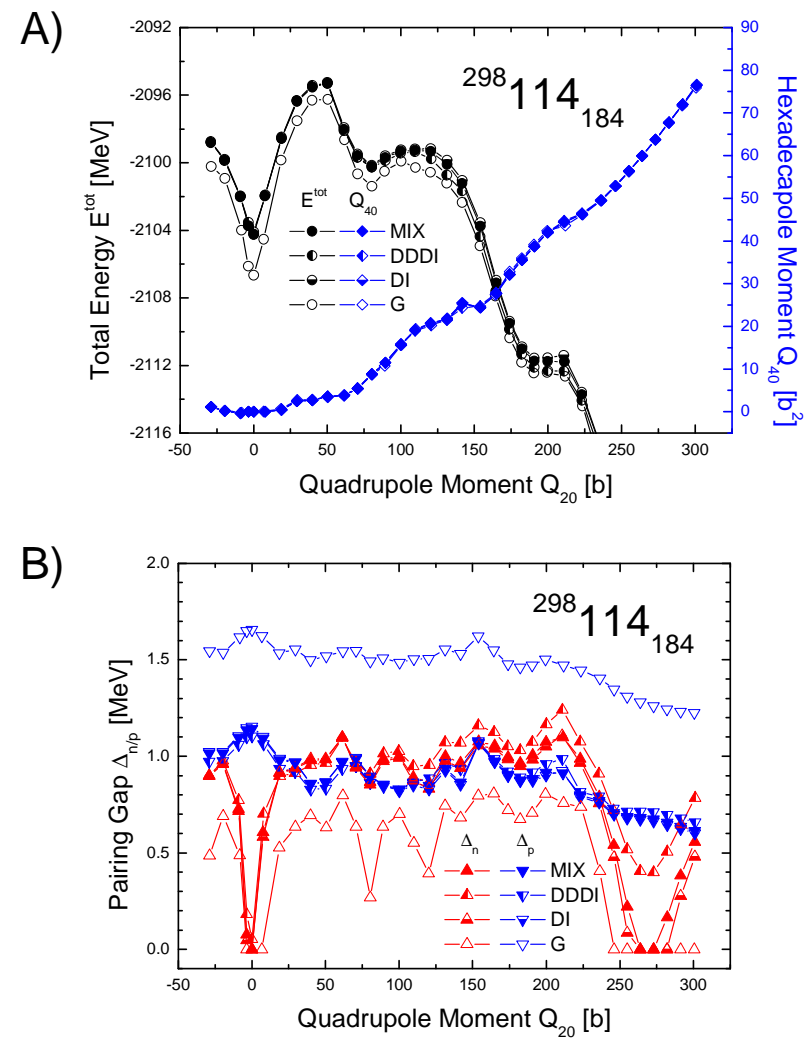

Fig. 2. Same as in Fig. 1 but now for ${ }^{298} 114_{184}$.

of $\Delta_{p}$ are considerably reduced. As expected, proton pairing is much weakened for $Z=126$ which is predicted to be semi-magic.21|22|23

The calculated static fission paths have reflection-symmetric shapes, i.e., $Q_{\lambda 0}=$ 0 for all odd multipolarities $\lambda$. Furthermore, one can see that irrespective of the pairing interaction used, the hexadecapole moments are almost identical, and they gradually increase (from 0 up to about $80 \mathrm{~b}^{2}$ ) along the calculated static fission paths. In contrast to $Q_{40}$, the collective potentials (i.e., total energies $E^{\text {tot }}$ as functions of $Q_{20}$ ) differ depending on the pairing model employed. This is particularly evident for ${ }^{288} \mathrm{Rf}_{184}$ (Fig. 1A ) where the fission barrier calculated within the $\mathrm{SHF}+\mathrm{BCS}(\mathrm{G})$ model is significantly higher as compared to those obtained in the $\mathrm{SHF}+\mathrm{BCS}(\delta)$ variants. In the case of ${ }^{298} 114_{184}$ (Fig. 22A), the fission barriers calculated with the $\mathrm{SHF}+\mathrm{BCS}(\mathrm{G})$ and $\mathrm{SHF}+\mathrm{BCS}(\delta)$ interactions are considerably closer to one another than in the case of ${ }^{288} \mathrm{Rf}_{184}$. Furthermore, for ${ }^{310} 126_{184}$ (Fig. 3A), all fission barriers calculated with both pairing models are almost identical. This can be attributed to the behavior of proton pairing along the fission paths. Indeed, in ${ }^{288} \mathrm{Rf}_{184}$ there is a large systematic difference between $\Delta_{p}$ values in $\mathrm{SHF}+\mathrm{BCS}(\mathrm{G})$ 
A)

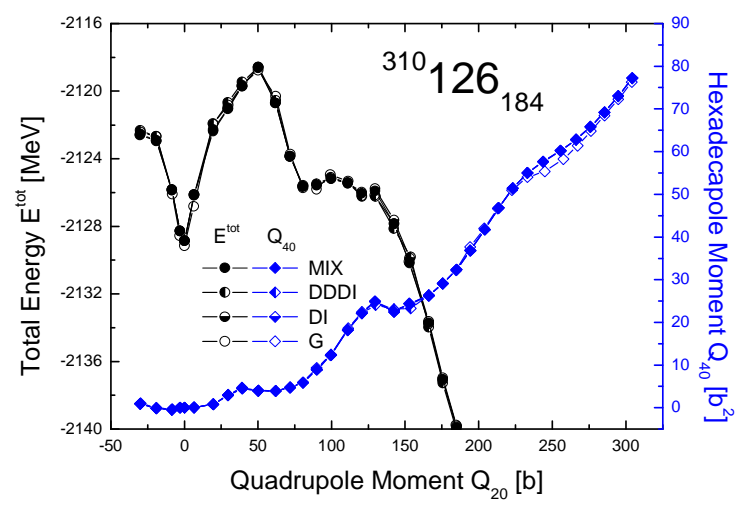

B)

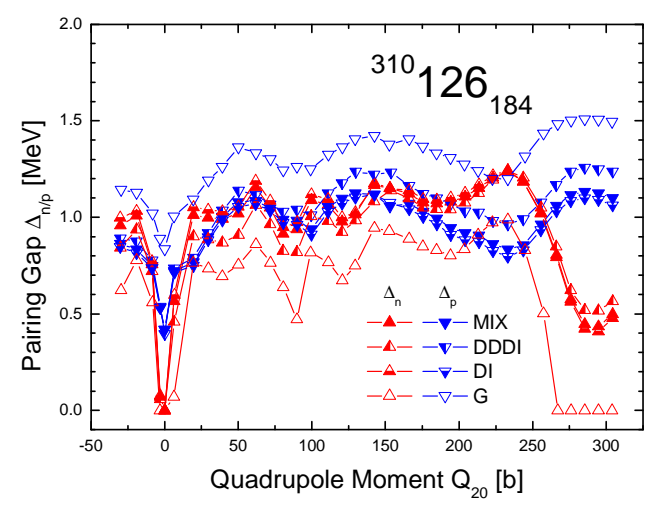

Fig. 3. Same as in Fig. 1 but now for ${ }^{310} 126184$.

and $\mathrm{SHF}+\mathrm{BCS}(\delta)$ variants, with the seniority-pairing model producing considerably larger pairing gaps. This difference decreases when going towards ${ }^{310} 126_{184}$ in which $\Delta_{n / p}$ obtained within the $\mathrm{SHF}+\mathrm{BCS}(\mathrm{G})$ model are much closer to those obtained within the $\mathrm{SHF}+\mathrm{BCS}(\delta)$ model. This result indicates that the isospin dependence of seniority pairing strengths given by Eq. (11) is too strong and thus unrealistic. Another interesting observation is that neutron/proton pairing gaps (hence, corresponding potential energies) calculated within the $\mathrm{SHF}+\mathrm{BCS}(\delta)$ framework are very similar, regardless of the parameterization variant used (MIX, DDDI or DI), see Eq. (4).

\subsection{Fission barriers for the even-even $N=184$ isotones with $\delta$-pairing interaction}

Figure 4 compares the total binding energies $\left(E^{\text {tot }}\right)$ and the mass hexadecapole moments $\left(Q_{40}\right)$ calculated along the fission paths for twelve even-even $N=184$ isotones. Here we use the MIX parameterization of the $\delta$-interaction. We found that all of the super-heavy elements (SHE) studied here have reflection-symmetric static fis- 

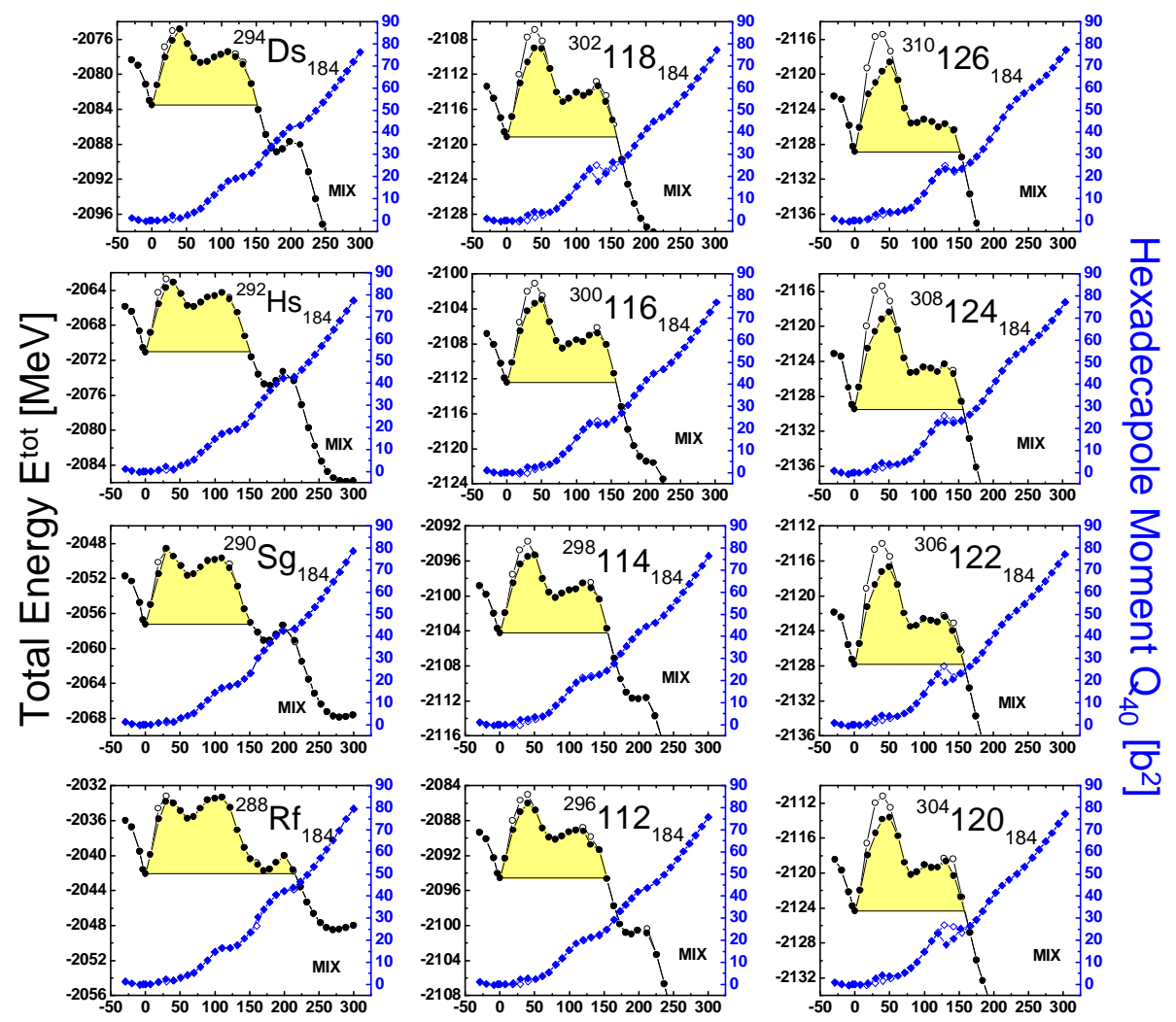

\section{Quadrupole Moment $\mathrm{Q}_{20}[\mathrm{~b}]$}

Fig. 4. The total binding energies $E^{\text {tot }}(\bullet$ - scales on the left-hand side) and mass hexadecapole moments $Q_{40}(\checkmark$ - scales on the right-hand side) along the fission paths, calculated with the SLy4 interaction and the MIX parameterization of the $\delta$-interaction for the even-even $N=184$ isotones of $288 \leq A \leq 310$. The differences between open and solid symbols illustrate the effects of triaxiality on the inner barriers.

sion paths and are spherical in the ground states, and the $Q_{40}$ moments calculated along the static fission paths follow the same pattern; i.e., their values continuously increase from 0 up to about $80 \mathrm{~b}^{2}$.

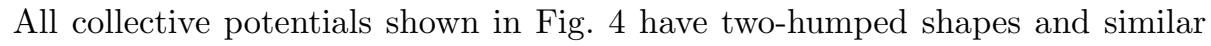
widths. Only in the case of ${ }^{288} \mathrm{Rf}_{184}$, can one see an additional small third external barrier. The outer barrier is systematically reduced with $Z$. The differences between open and solid symbols illustrate the effect of triaxiality on the inner barriers. The strongest effect is predicted for the nucleus ${ }^{310} 126_{184}$, where the barrier is lowered by more than $3 \mathrm{MeV}$ due to triaxiality. However, for lighter isotones with $Z \leq 114$, the role of triaxiality is modest.

For completeness, it is interesting to look at the neutron $\left\langle\Delta_{n}\right\rangle$ and proton $\left\langle\Delta_{p}\right\rangle$ spectral pairing gaps calculated along the static fission paths shown in Fig. 4. They 


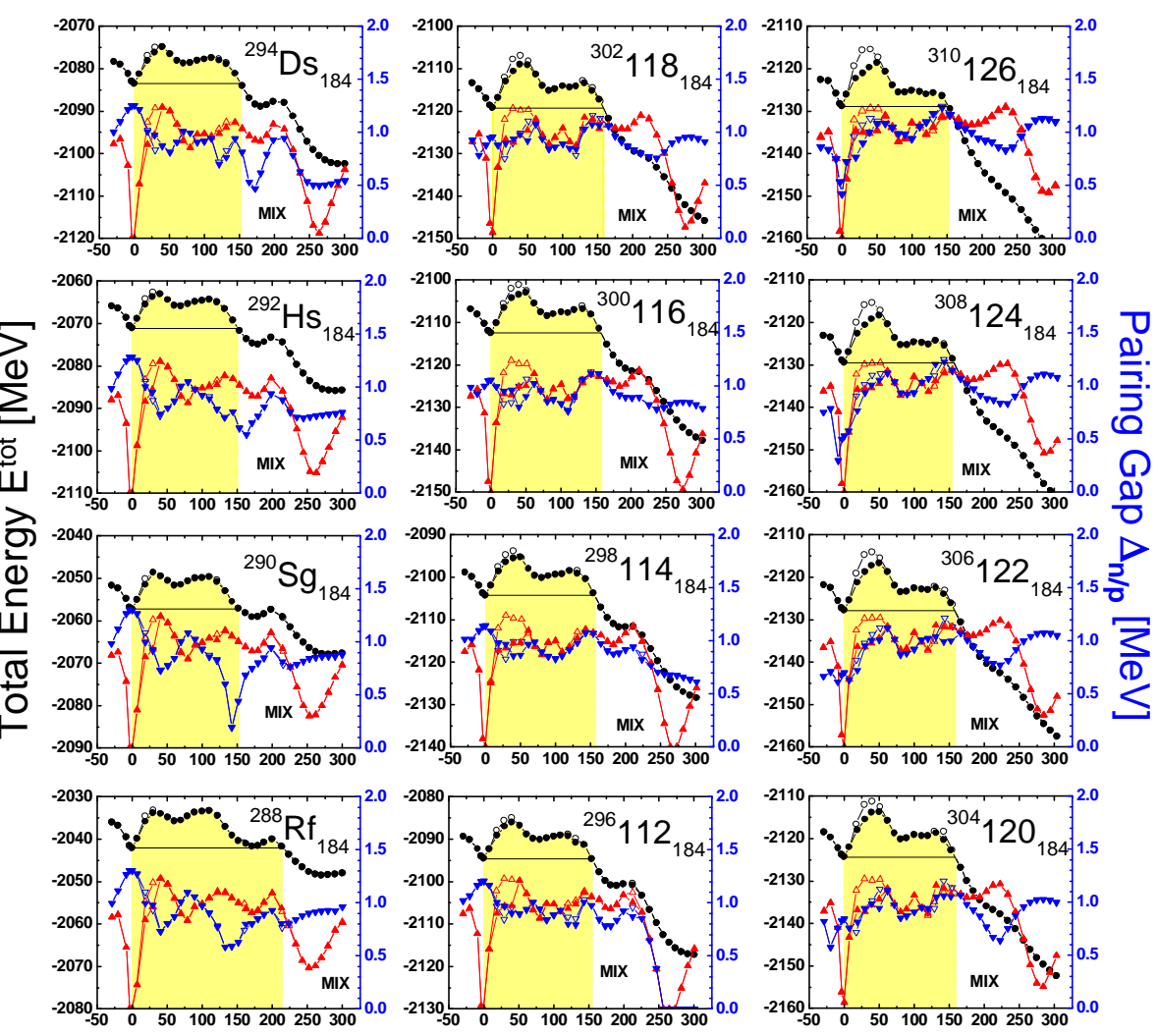

Quadrupole Moment $\mathrm{Q}_{20}[\mathrm{~b}]$

Fig. 5. The total binding energies $E^{\text {tot }}(\bullet$, scales on the left-hand side) and the spectral neutron $\left\langle\Delta_{n}\right\rangle(\boldsymbol{\Lambda})$ and proton $\left\langle\Delta_{p}\right\rangle(\mathbf{\nabla})$ pairing gaps (scales on the right-hand side) for the $N=184$ isotones shown in Fig. 4

are shown in Fig. 5. As already mentioned, the ground-state neutron pairing gaps $\left\langle\Delta_{n}\right\rangle(\mathbf{\Lambda})$ are equal to 0 in all the $N=184$ isotones, whereas the proton gaps $\left\langle\Delta_{p}\right\rangle$ $(\mathbf{v})$ change from $1.3 \mathrm{MeV}$ in ${ }^{288} \mathrm{Rf}_{184}$ ) to less than $0.5 \mathrm{MeV}$ in ${ }^{288} 126_{184}$. In the sub-barrier regions of the fission paths (shadowed regions in the plot), neutron and proton spectral pairing gaps fluctuate around $1 \mathrm{MeV}$.

The size of a fission barrier is a measure of stability of the nucleus against spontaneous fission. The static fission barriers shown in Fig. 4 1 suggest a possible increase of the stability against spontaneous fission for $118 \leq Z \leq 124$. This predicted increase of the stability supports the results obtained in our previous paper $\frac{10}{10}$ where the same nuclei were considered within the $\mathrm{SHF}+\mathrm{BCS}(\mathrm{G})$ framework, but with the pairing strengths of Eq. (1) scaled to reproduce the pairing gaps of the finite-range droplet model (FRDM) 24 


\section{Conclusion}

The purpose of this work was to study the sensitivity of static fission trajectories in $N=184$ isotones to the choice of pairing interaction in the SHF+BCS model. We found that the results (energies, hexadecapole moments, pairing gaps) are fairly insensitive to the assumed density dependence of the pairing $\delta$-interaction (DI, DDDI, or MIX). On the other hand, we found rather large differences between results obtained in $\delta$-pairing and seniority-pairing variants. This suggests that the isospin dependence of the seniority-pairing strength used is unrealistic. In general, it is always advantageous to consider a more realistic interaction in the pairing channel (such as the density-dependent $\delta$ force) which does not depend explicitly on mass number and neutron excess.

In the second part of the paper, we examined static fission paths calculated within the $\mathrm{SHF}+\mathrm{BCS}(\delta)$ framework and MIX pairing parameterization for twelve even-even $N=184$ isotones. We found that the reduction of fission barriers due to the appearance of triaxial deformation strongly depends on $Z$. Furthermore, the analysis of the static fission barriers suggests the increased stability against spontaneous fission for $118 \leq Z \leq 124$.

\section{Acknowledgements}

This work was supported in part by the National Nuclear Security Administration under the Stewardship Science Academic Alliances program through the U.S. Department of Energy Research Grant DE-FG03-03NA00083; by the U.S. Department of Energy under Contract Nos. DE-FG02-96ER40963 (University of Tennessee), DE-AC05-00OR22725 with UT-Battelle, LLC (Oak Ridge National Laboratory), and DE-FG05-87ER40361 (Joint Institute for Heavy Ion Research); by the Polish Committee for Scientific Research (KBN) under Contract No. 1 P03B 059 27; by

the Foundation for Polish Science (FNP); and by the Polish Ministry of Science and Higher Education under Contract No. N202 179 31/3920.

\section{References}

1. A. Bohr, B. R. Mottelson, and D. Pines, Phys. Rev. 110, 936 (1958).

2. L. N. Cooper, R. L. Mills, and A. M. Sessler, Phys. Rev. 114, 1377 (1959).

3. S. Belyaev, Mat. Fys. Medd. Dan. Vid. Selsk. 31, 641 (1959).

4. D.J. Dean and M. Hjorth-Jensen, Rev. Mod. Phys. 75, 607 (2003).

5. E. Perlińska, S.G. Rohoziński, J. Dobaczewski, and W. Nazarewicz, Phys. Rev. C 69, 014316 (2004).

6. D.M. Brink and R.A. Broglia, Nuclear Superfluidity: Pairing in Finite Systems (Cambridge University Press, 2005).

7. F. Barranco, P. F. Bortignon, R.A. Broglia, G. Coló, P. Schuck, E. Vigezzi, and X. Viñas, Phys. Rev. C 72, 054314 (2005).

8. M. Bender, P.-H. Heenen, and P.-G. Reinhard, Rev. Mod. Phys. 75, 121 (2003).

9. W. Nazarewicz, Nucl. Phys. A 557, 489c (1993).

10. A. Staszczak, J. Dobaczewski, and W. Nazarewicz, Int. J. Mod. Phys. E15, 302-310 (2006). 
11. E. Chabanat, P. Bonche, P. Haensel, J. Meyer, and F. Schaeffer, Nucl. Phys. A635, 231 (1998); A643, 441(E) (1998).

12. J. Dudek, A. Majhofer, and J. Skalski, J. Phys. G6, 447 (1980).

13. R. R. Chasman, Phys. Rev. C 14, 1935 (1976).

14. J. Dobaczewski, W. Nazarewicz, and M.V. Stoitsov, Eur. Phys. J. A 15, 21 (2002).

15. G. Audi, A. H. Wapstra, and C. Thibault, Nucl. Phys. A729, 337-676 (2003); http://csnwww.in2p3.fr/amdc/masstables/Ame2003/rct7.mas03.

16. M. Bender, K. Rutz, P.-G. Reinhard, and J. A. Maruhn, Eur. Phys. J. A8, 59 (2000).

17. J. Dobaczewski and J. Dudek, Comput. Phys. Commun. 102, 166-182 (1997); ibid. 102, 183-209 (1997); ibid. 131, 164-186 (2000).

18. J. Dobaczewski and P. Olbratowski, Comput. Phys. Commun. 158, 158-191 (2004); ibid. 167, 214-216 (2005).

19. J. Dobaczewski et al., to be published in Comput. Phys. Commun.; HFODD home page: http://www.fuw.edu.pl/ dobaczew/hfodd/hfodd.html.

20. H. Flocard, P. Quentin, A. K. Kerman, and D. Vautherin, Nucl. Phys. A203, 433-472 (1973).

21. S. Ćwiok, J. Dobaczewski, P.-H. Heenen, P. Magierski, and W. Nazarewicz, Nucl. Phys. A 611, 211 (1996).

22. S. Ćwiok, P.H. Heenen, and W. Nazarewicz, Nature 433, 705 (2005).

23. M. Bender, W. Nazarewicz, and P.-G. Reinhard, Phys. Lett. B 515, 42 (2001).

24. P. Möller, J. R. Nix, and K.-L. Kratz, At. Data Nucl. Data Tables 66, 131-343 (1997); http://t2.lanl.gov/data/astro/molnix96/pairlip.dat. 